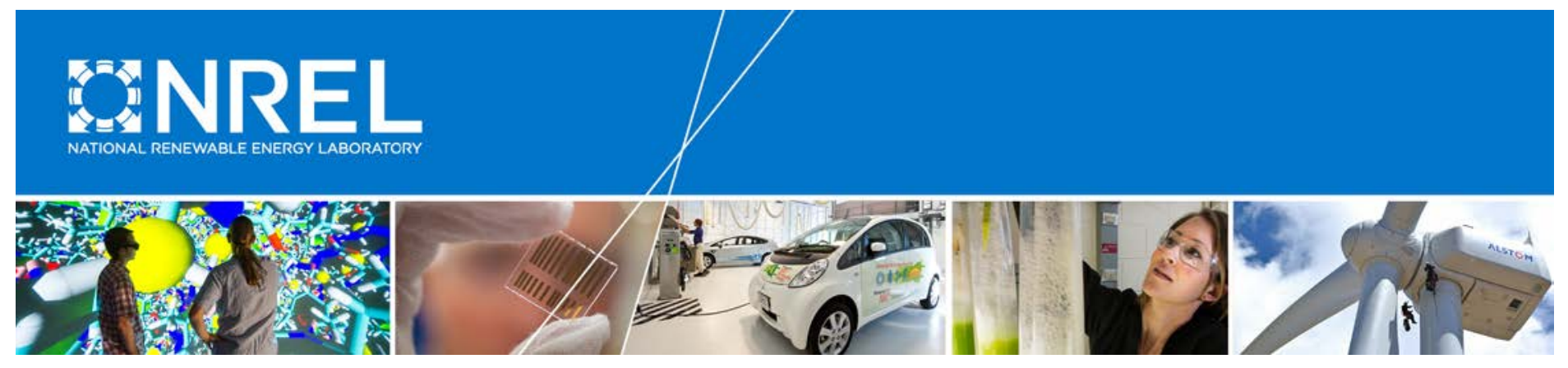

\title{
A New Approach for Short-Term Solar Radiation Forecasting Using the Estimation of Cloud Fraction and Cloud Albedo
}

Andrew Kumler, Yu Xie, and Yingchen Zhang National Renewable Energy Laboratory

NREL is a national laboratory of the U.S. Department of Energy Office of Energy Efficiency \& Renewable Energy Operated by the Alliance for Sustainable Energy, LLC

This report is available at no cost from the National Renewable Energy Laboratory (NREL) at www.nrel.gov/publications.

Technical Report

NREL/TP-5D00-72290

October 2018 


\title{
A New Approach for Short- Term Solar Radiation Forecasting Using the Estimation of Cloud Fraction and Cloud Albedo
}

\author{
Andrew Kumler, Yu Xie, and Yingchen Zhang \\ National Renewable Energy Laboratory \\ Suggested Citation \\ Kumler, Andrew, Yu Xie, and Yingchen Zhang. 2018. A New Approach for \\ Short-Term Solar Radiation Forecasting Using the Estimation of Cloud \\ Fraction and Cloud Albedo. Golden, CO: National Renewable Energy \\ Laboratory. NREL/TP-5D00-72290. \\ https://www.nrel.gov/docs/fy19osti/72290.pdf.
}

NREL is a national laboratory of the U.S. Department of Energy Office of Energy Efficiency \& Renewable Energy Operated by the Alliance for Sustainable Energy, LLC

This report is available at no cost from the National Renewable Energy Laboratory (NREL) at www.nrel.gov/publications.

National Renewable Energy Laboratory 15013 Denver West Parkway Golden, CO 80401

303-275-3000 • www.nrel.gov

\section{Technical Report}

NREL/TP-5D00-72290

October 2018

Contract No. DE-AC36-08GO28308 


\section{NOTICE}

This work was authored by the National Renewable Energy Laboratory, operated by Alliance for Sustainable Energy, LLC, for the U.S. Department of Energy (DOE) under Contract No. DE-AC36-08G028308. Funding provided by U.S. Department of Energy Office of Energy Efficiency and Renewable Energy Solar Energy Technologies Office. The views expressed in the article do not necessarily represent the views of the DOE or the U.S. Government.

This report is available at no cost from the National Renewable Energy Laboratory (NREL) at www.nrel.gov/publications.

U.S. Department of Energy (DOE) reports produced after 1991 and a growing number of pre-1991 documents are available free via www.OSTI.gov.

Cover Photos by Dennis Schroeder: (left to right) NREL 26173, NREL 18302, NREL 19758, NREL 29642, NREL 19795.

NREL prints on paper that contains recycled content. 


\section{List of Acronyms}

DNI

GHI

MAE

MBE

nRMSE

NWP

PSPI

RMSE

SZA
Direct normal irradiance

Global horizontal irradiance

Mean absolute error

Mean bias error

Normalized root mean square error

Numerical Weather Prediction

Physics-Based Smart Persistence Model for Intra-Hour Solar Forecasting Root mean square error

Solar zenith angle 


\section{Executive Summary}

Solar generation is an increasing part of the energy portfolio in the United States. An accurate forecast of the available solar resource and power is essential to managing the electric grid, market operations, and reducing the cost of solar energy. High-frequency forecasts of solar radiation in intra-hour horizons is important for real-time electric power system energy management, especially at the distribution level. Conventional Numerical Weather Prediction models perform poorly in intra-hour, high-frequency forecasts because of the limits on real-time computing, spatial resolution, and infrequent availability of observations. Although a number of alternative technologies, e.g., time-series analysis and machine learning, have been used to fill this gap, the smart persistence model is among the top-performing models in short-term forecasting and therefore often serves as the baseline to evaluate other forecasting models.

Although the smart persistence model often serves as the baseline model in these intra-hour forecasts, obvious uncertainties exist in the current smart persistence model: (1) clear-sky index does not respond to the variation of the solar incident angle when cloud conditions are persistent within the forecast horizon, and (2) cloud coverage is inherently persistent though it is constrained by cloud advection. In this study, we developed a Physics-Based Smart Persistence Model for Intra-Hour Solar Forecasting (PSPI) that integrates cloudy property estimation, a radiative transfer model, and cloud fraction forecasts to improve the performance of the smart persistence model. Compared to the smart persistence model, PSPI does not require additional observations of various atmospheric parameters past global horizontal irradiance, but it is customizable because additional observations, if available, can be ingested to further improve the forecast. Our results show that the PSPI outperforms the persistence and smart persistence model on 5-minute, 15-minute, and 30-minute forecast horizons. The software package of PSPI is flexible to users' needs and provides low computational time to run at site-specific locations across the continental United States. 


\section{Table of Contents}

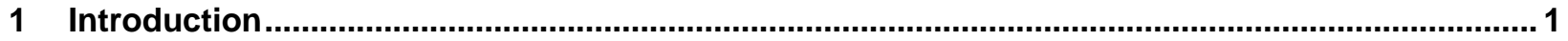

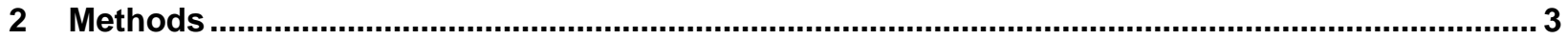

2.1 Review of Persistence Models ..................................................................................... 3

2.2 Simulation of GHI Using Estimations of Cloud Fraction and Cloud Albedo ........................... 3

2.3 An Improved Smart Persistence Model for Short-Term Solar Forecasting ...............................4

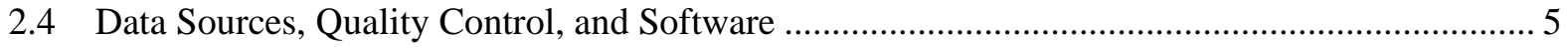

2.5 Metrics for Evaluating Model Performance ............................................................ 5

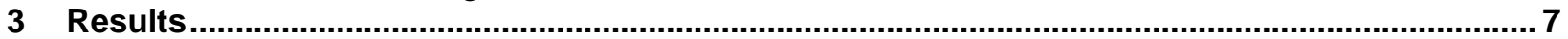

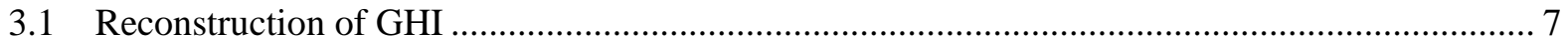

3.2 Performance of the PSPI model .............................................................................. 7

4 Conclusions ........................................................................................................................ 12

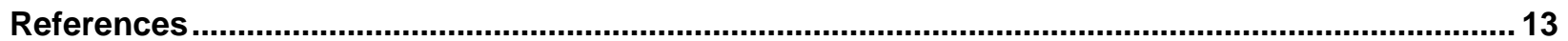




\section{List of Figures}

Figure 1. Comparison of observations versus reconstruction for the clear-sky day of July 17, 2013 (top) and the cloudy-sky day of December 2, 2013 (bottom)......................................................... 7

Figure 2. 5-minute (top), 15-minute (middle), and 30-minute forecast (bottom) for the smart persistence and PSPI models for the cloudy-sky day of December 2, 2013........................................... 9

Figure 3. Clearness-cloud fraction bins for the 5-minute (left), 15-minute (middle), and 30-minute (right)

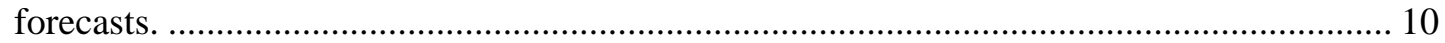

Figure 4. Taylor diagram showing the performance of the 5-minute (left), 15-minute (middle), and 30minute (right) forecasts for the year 2013.................................................................... 10

\section{List of Tables}

Table 1. Annual Statistics (2013) for the 5-Minute Forecast................................................................. 11

Table 2. Annual Statistics (2013) for the 15-Minute Forecast............................................................... 11

Table 3. Annual Statistics (2013) for the 30-Minute Forecast.................................................................. 11 


\section{Introduction}

Each year, solar energy is increasingly integrated into the electric grid. Costumer adoption of small distributed PVs such as rooftop has outpaced the growth of utility scale PVs, and they are expected to compose of 50\%-60\% of total U.S. PV capacity through 2020 (Palmintier 2016). Electrical utilities are heavily investing in Distribution Management Systems (DMS) technologies to increase situational awareness at the distribution level and enable an efficient, reliable, and secure control of highly distributed energy assets. With the rapid increase in distributed solar levels, the DMS systems will require accurate forecasts of PV patterns/variability and will need to ensure that control schemes offer centralized and distributed decision-making opportunities that match the time scales of ambient conditions (Kaur et al. 2016; Mills 2013). For decades, solar forecasting methods have been studied in the literature using a variety of techniques and for a plethora of forecast time horizons. Thorough reviews of solar forecasting techniques can be found in Inman, Pedro, and Coimbra et al. (2013) and Sengupta et al. (2017). Of these solar forecasting techniques, time-series models are among the most original and simplest (Goh and Tan 1977). Time-series models often use solar radiation measurements, such as global horizontal irradiance (GHI), in addition to statistical models and possibly simple atmospheric radiation physics (Kleissl 2013). These models usually operate in the intra-hour forecast horizon, with more complex solar forecasting methods being more accurate past 1 hour, e.g., satellite-based methods, Numerical Weather Prediction (NWP). With current technologies (computing power, access to observations, etc.) NWP at the intra-hour forecast horizon is extremely expensive and not practical, especially for the renewable energy sector. The focus of traditional uses for NWP forecasts has been on severe weather and aviation, with renewable energy forecasts only recently becoming a topic of interest and study (Haupt et al. 2016; Haupt et al. 2018; Mathiesen and Kleissl 2011; Perez et al. 2013). To help fill this gap, techniques such as machine learning and time-series analysis, using multiple forecast horizons and data sets, have become popular in the literature (Inman, Pedro, and Coimbra 2013; Yang et al. 2018).

The intra-hour forecast horizon is interesting from both an atmospheric science and an energy management perspective. From the atmospheric science perspective, the most interesting cloud phenomena happen sub hourly, with changes in cloud conditions having large impacts on incident solar radiation. From the energy management side, especially for solar energy, the quick changes in incident solar radiation can drastically alter the production of solar power especially in distribution systems with high penetrations of solar (Denholm and Hand 2011). To help alleviate this problem, time-series models can help detect future intra-hour ramps of solar irradiance and thus power. The most common of these models are the persistence and smart persistence models (see Section 2.1). These models are the simplest time-series models used in the solar energy community, and they are often used as a baseline to compare other forecast models. These qualities make them attractive for use by utilities. In addition, they need little to no observations of GHI and limited supervision, making them a cost-effective solution. Despite this, the smart persistence model suffers from two main problems: (1) the inability for the incident solar angle to change when cloud conditions are persistent, and 2) cloud cover remains persistent but is eventually advected depending on the forecast horizon. These two problems are also dependent on how often the smart persistence model is updated (e.g., 1-minute, 5-minute, 
30-minute). These pitfalls of the smart persistence model and the need for a simple yet robust intra-hour solar irradiance forecast are the main motivations for this study.

The purpose of this paper is to introduce a Physical Smart Persistence Model (PSPI) that is capable of better capturing cloud conditions than typical persistence models while needing only local GHI observations to run. In addition, the code that runs the model is customizable because additional atmospheric observations, if available, can be easily ingested into the code and thus produce a more realistic forecast. Last, utilities and grid operators can take advantage of PSPI by converting an irradiance forecast into an intra-hour power forecast, depending on their needs. Increased number of utilities' installation of local solar irradiance sensors provide foundation of the proposed method to be widely adopted (Kuszamaul et al. 2010).

The rest of this paper discusses the methods on which the PSPI model is built and how it operates (Section 2); results and performance of the PSPI model against traditional persistence models (Section 3); and conclusions that can be drawn from using the PSPI, including potential benefits to utilities and energy management systems (Section 4). Future work for improving the model is also considered. 


\section{Methods}

\subsection{Review of Persistence Models}

In solar forecasting, the persistence model has often served as the baseline model to compare other models. Even today, the persistence model (and smart persistence model described next) performs well for intra-hour forecasts, mainly because accurately capturing cloud conditions remains elusive (Kleissl 2013; Marquez and Coimbra 2013). The simple persistence model can be defined as:

$$
I_{p}(t+\Delta t)=I(t)
$$

where $I(t)$ is the current irradiance (GHI) at the surface. The persistence model can be improved by adding a clear-sky index correction factor, which is the ratio of GHI measurements divided by clear-sky GHI (Kaur et al. 2016, Sengupta et al. 2017). This clear-sky index is then multiplied by future clear-sky GHI to obtain a forecast, defined as:

$$
I_{s p}(t+\Delta t)=k_{t}(t) * I_{c l r}(t+\Delta t)
$$

where $k_{t}(t)$ is the clear-sky index, and $I_{c l r}(t+\Delta t)$ is the clear-sky GHI in a future time step. The benefit of the smart persistence model is that general atmospheric conditions are indirectly observed through the clear-sky index and advected into some future time. The clear-sky GHI can be computed through a variety of clear-sky models, each with varying degrees of complexity and accuracy (Ineichen 2016).

\subsection{Simulation of GHI Using Estimations of Cloud Fraction and Cloud Albedo}

A major drawback to using a persistence model is that atmospheric conditions (mainly cloud conditions) are largely unknown. These conditions are hidden in the persistent GHI and are advected to a future forecast. Following the approach of retrieving cloud albedo and cloud fraction from Xie and Liu (2013), GHI can be reconstructed for the current time period while obtaining cloud conditions. To begin, certain irradiance variables are required to complete the reconstruction. These include clear-sky GHI at the surface (hereafter $F_{c l r}^{d n}$ ), clear-sky direct normal irradiance (DNI) at the surface (hereafter $F_{c l r, d}^{d n}$ ), GHI measured at the surface by a CMP22 (Kipp and Zonen pyranometer, hereafter $F_{\text {all }}^{d n}$ ), all-sky DNI at the surface (hereafter $F_{a l l, d}^{d n}$ ), and all-sky upward GHI (hereafter $F_{\text {all }}^{u p}$ ). $F_{\text {all }}^{u p}$ is given by the following equation:

$$
F_{\text {all }}^{u p}=\alpha_{s} F_{\text {all }}^{d n}
$$

where $\alpha_{s}$ is the land surface albedo, assumed to be 0.2 in this study. These variables can be obtained using the following methods: $F_{c l r}^{d n}$ and $F_{c l r, d}^{d n}$ can be modeled using a clear-sky model, and $F_{a l l, d}^{d n}$ can be modeled using a DNI model. We chose the Ineichen-Perez clear-sky model (Ineichen and Perez 2002) because of its great performance among other clear-sky models while needing only minimal input (Reno, Hansen, and Stein 2012). For DNI, we chose the Erbs model (Erbs, Klein, and Duffie 1982) mainly because of its simplicity and speed. 
Once these variables are obtained, reconstruction of current GHI can begin. As laid out in Xie and Liu (2013), GHI at the surface for a single-layer cloud atmosphere can be expressed as:

$$
F_{\text {recon }}^{\text {dn }}=F_{1}\left(1-\alpha_{s} \alpha_{r} f T^{2}\right)^{-1}
$$

where $\alpha_{s}$ is the surface albedo, $\alpha_{r}$ is the cloud albedo, $f$ is the cloud fraction, $\mathrm{T}$ is the transmittance of diffuse radiation of the atmosphere, and $F_{1}$ is the first-order downwelling flux at the surface. $F_{1}$ is given by:

$$
F_{1}=f F_{c l d}^{d n}+(1-f) F_{c l r}^{d n}
$$

where $F_{c l d}^{d n}$ is the downwelling flux because of cloudy skies, and it can be approximated by:

$$
F_{c l d}^{d n}=\left(1-\alpha_{r}\right) F_{c l r}^{d n}
$$

with $F_{c l r}^{d n}$ being the clear-sky GHI that was obtained from the Ineichen-Perez model. Other parameters here, such as $\alpha_{r}$ and $f$, are explained in greater detail in Xie and Liu (2013).

\subsection{An Improved Smart Persistence Model for Short-Term Solar Forecasting}

With the ability to reconstruct GHI using the method outlined in the previous section, it is then possible to forecast GHI using the same variables. In the future time step within 0-30 minutes, we assume semi-persistent cloud optical thickness and cloud fraction. Cloud thickness and fraction can be updated as often as desired, depending on the user's needs and computing abilities. For this study, cloud fraction is semi-persistent for a given forecast horizon (e.g., a 15minute forecast assumes cloud conditions remain unchanged from 15 minutes prior) but is computed as a 5-minute rolling average. This changes if original cloud fraction values (nonrolling average) ramp up or down within a 5-minute moving window. If cloud fraction changes by more than $30 \%$ in a 5-minute window, the forecast changes to a 5-minute forecast until cloud conditions stabilize. The original forecast is modified to reflect changing cloud conditions in addition to making a normal (e.g., 15-minute or 30-minute) forecast. The cloud albedo remains largely persistent but is also updated when the forecast changes to 5 minutes. Cloud albedo can be given by a two-stream approximation suggested by Sagan and Pollack (1967) when cloud absorption is neglected (Meador and Weaver 1980):

$$
\alpha_{r}^{\prime}=\frac{b \tau / \mu_{0}^{\prime}}{1+b \tau / \mu_{0}^{\prime}}
$$

where $\tau$ is the cloud optical thickness, defined as:

$$
\tau=\frac{2 \alpha_{r} \mu_{0}}{\left(1-\alpha_{r}\right)(1-g)}
$$

with $\mu_{0}=|\cos (\mathrm{SZA})|$ (SZA being the solar zenith angle), $\mathrm{g}$ is the asymmetry factor of cloud particles assumed to be 0.86, and $b=0.05-0.05 \mathrm{~g}$ (Xie and Liu 2013). The prime denoted on 
some of the variables indicates values for the future time step. Following these equations and from Section 2.2, the forecast for GHI can be given by:

$$
\begin{aligned}
& F_{a l l}^{d n^{\prime}}=F_{1}^{\prime}\left(1-\alpha_{s} \alpha_{r}^{\prime} f T^{2}\right)^{-1} \\
& F_{1}^{\prime}=f F_{c l d}^{d n^{\prime}}+(1-f) F_{c l r}^{d n^{\prime}} \\
& F_{c l d}^{d n^{\prime}}=\left(1-\alpha_{r}^{\prime}\right) F_{c l r}^{d n^{\prime}}
\end{aligned}
$$

where $F_{c l r}^{d n '}$ can be computed by the Ineichen-Perez model and the solar zenith angle by a variety of Python libraries (see next section).

\subsection{Data Sources, Quality Control, and Software}

The measurement data used in this study are available for download from the National Renewable Energy Laboratory Solar Radiation Research Laboratory - Measurement and Instrument Data Center (www.midcdmz.nrel.gov). The Solar Radiation Research Laboratory is located in Golden, Colorado, at an elevation 1,829 m above sea level, with atmospheric influences from the Rocky Mountains and the urban environment of the Denver Metro area, creating a complex site to model. Here, data can be downloaded or viewed in real time. It also contains historical data dating to 1981 (Stoffel and Andreas 1981). Most data come quality controlled, so little post-processing is needed. The forecast data generated from the equations in Section 2.3, and thus observations from the Solar Radiation Research Laboratory, undergo some simple quality control, such as eliminating data that occur when the solar elevation angle is less than $7^{\circ}$ (Ruiz-Arias et al. 2010), correcting modeled values that exceed extraterrestrial radiation at any point in the time series, and eliminating the occasional (but rare) erratic value that differs unreasonably from observations. Some of these bad data situations can occur because of the inherent simplicity of the model. Many atmospheric parameters are assumed to be constant (precipitable water, aerosol content, pressure, etc.), which can lead to under- or overestimations of clear-sky variables, which thus produces instances of unphysical cloud variables. Although a reasonable forecast can still be made despite this, this hinders the ability to accurately know cloud conditions. To help alleviate this, measures can be taken to better capture the current state of the atmosphere, such as ingesting more data sources (if available) and setting upper and lower thresholds.

To assist the forecast model with various variables such as extraterrestrial irradiance and clearsky GHI, the open-source and community-supported PVLIB Python library is used (Holmgren et al. 2015, 1-5). PVLIB is a great tool for assisting and enhancing solar resource and forecasting studies, with common tools and models found in the literature aggregated into one place.

\subsection{Metrics for Evaluating Model Performance}

To verify how well models explained in the previous sections perform against observations, various statistical measures common in the solar forecasting community can be used (Inman et al. 2013; Sengupta et al. 2017). One of the most common and straightforward statistical methods is the Pearson correlation (Benesty et al. 2009, 1-4), which measures the linear relationship between two variables: 


$$
r=\frac{\sum_{i=1}^{n}\left(m_{i}-\bar{m}\right)\left(o_{i}-\bar{o}\right)}{\sigma(m) \sigma(o)}
$$

where $m$ represents the model value, and $o$ represents the observations. The Pearson correlation gives a very general idea of how well two data sets relate to each other. Other useful measures, such as mean bias error (MBE) and mean absolute error (MAE), give an idea of forecast accuracy. MBE helps identify if the model has a positive or negative bias, whereas MAE states the overall error of the model.

$$
\begin{aligned}
& M B E=\frac{1}{n} \sum_{i=1}^{n}\left(m_{i}-o_{i}\right) \\
& M A E=\frac{1}{n} \sum_{i=1}^{n}\left|m_{i}-o_{i}\right|
\end{aligned}
$$

One of the most common statistical metrics used in the solar forecasting community is the root mean square error (RMSE). It calculates the average spread of the error in a population, which is a good indicator of model performance:

$$
R M S E=\frac{1}{\sqrt{N}} \sqrt{\sum_{i=1}^{N}\left(m_{i}-o_{i}\right)^{2}}
$$

The last statistical metric used in this study is the forecast score (Marquez and Coimbra 2013; Yang et al. 2018). The forecast score takes the ratio of the normalized RMSE of a model over a persistence model and subtracts the results from one.

$$
F S=1-\frac{n R M S E_{\text {model }}}{n R M S E_{\text {persistence }}}
$$

This number indicates how well a model performs in relation to the persistence model (or model of choice), with a positive value indicating better performance and a negative value worse performance. Although many other statistical measures exist in the solar energy community (Gueymard 2014), we believe that the metrics outlined in this section give a well-rounded assessment of simple time-series models, such as persistence models. 


\section{Results}

\subsection{Reconstruction of GHI}

Before delving into the results of the PSPI model, it is first necessary to see how well the reconstruction of GHI matches observations. For all figures in this paper, results from the year 2013 are shown. Figure 1 shows the results of the reconstruction against observations for a clearsky day and a cloudy-sky day in 2013. As shown in each panel in Figure 1, reconstruction of GHI matches essentially perfectly with observations.
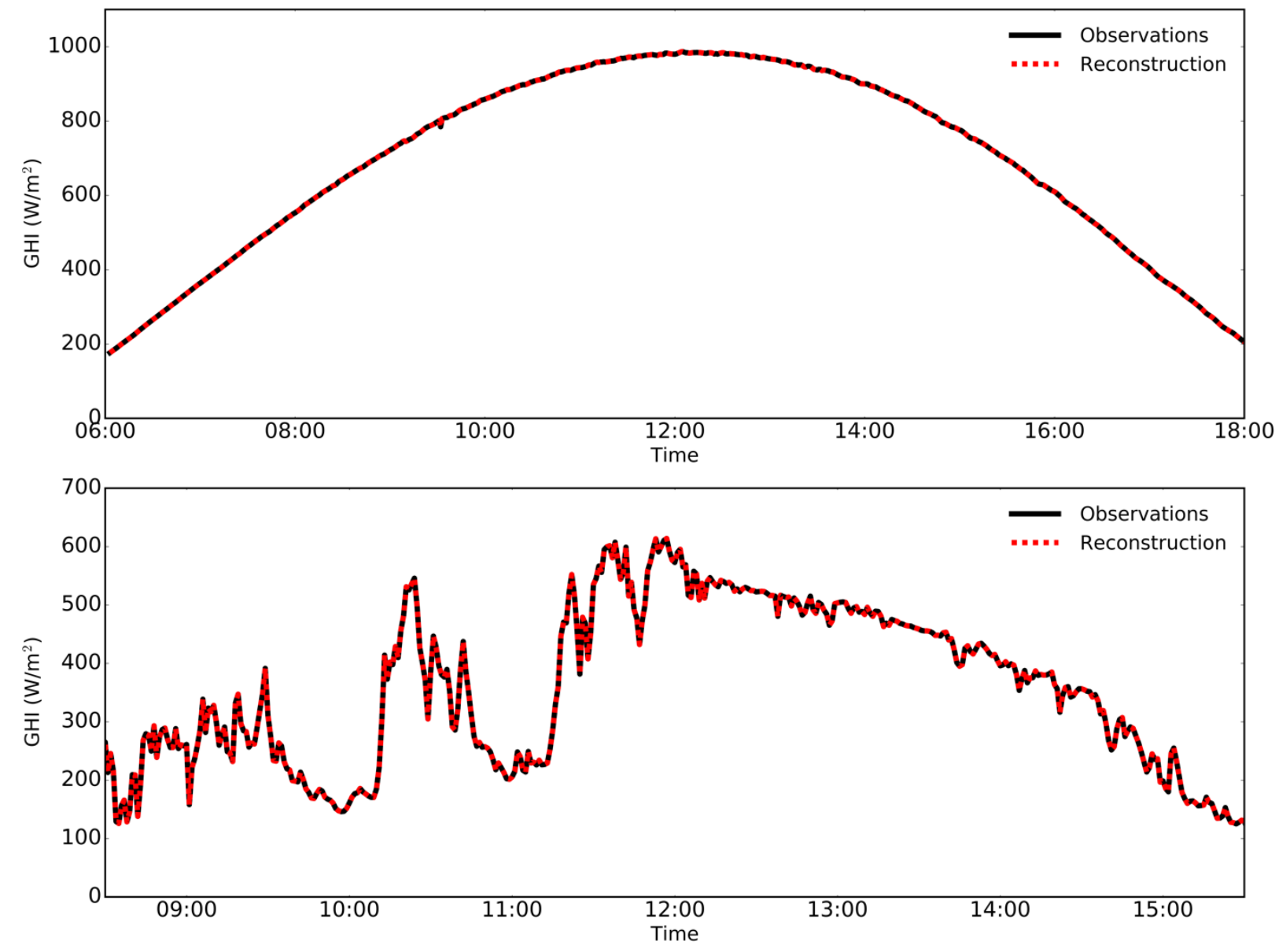

Figure 1. Comparison of observations versus reconstruction for the clear-sky day of July 17, 2013, (top) and the cloudy-sky day of December 2, 2013 (bottom)

\subsection{Performance of the PSPI model}

With the reconstruction of GHI being accurate, we can now evaluate the PSPI model. Figure 2 shows a time-series plot of a 5-minute, 15-minute, and 30-minute forecast for a cloudy-sky day in December 2013. As shown in this plot, especially the 15-minute and 30-minute forecasts, overall the PSPI has a better handle on cloud ramps than the smart persistence model. This is evident throughout the year under varying cloud conditions. In addition, as the forecast horizon 
decreases, the forecasts begin to converge, which is to be expected. This is also revealed in Figure 3, which bins the better performing model into clearness index and cloud fraction bins. For every combination of clearness index and cloud fraction, the better performing model at that time is assigned to that bin for the entirety of 2013. Referring to Section 2.4, if an unphysical cloud fraction value occurs, and to a lesser extent clearness index value, then this value is adjusted according to the theoretical maximum and minimum value possible for each respective variable. At the 30-minute forecast horizon (Figure 3, right panel), PSPI performs admirably in nearly all clearness index and cloud fraction situations. As the forecast horizon decreases, the forecasts become more similar until no clear difference can be made between the two models. Although smart persistence appears dominant in the 5-minute forecast horizon (Figure 3, left panel), the amount of area covered is not directly indicative of better performance. For example, most of the clearness index and cloud fraction combinations could happen in a particular range, which is not expressed in Figure 3. To address this, Figure 4 depicts three Taylor diagrams, one for each forecast horizon. Taylor diagrams (Taylor 2001) are a succinct way of showing three different statistics in one diagram and have generally been underappreciated in the solar energy community (Gueymard 2014). For these Taylor diagrams, the lower right corner (where correlation coefficient $=1$ and RMSD $=0$ ) is considered truth, or the statistics of the observations. The closer a model is to this point on the figure, and thus observations, the better the model is considered to be. As shown in Figure 4, for all forecast horizons, PSPI (red) outperforms both the smart persistence and persistence models (blue and green, respectively). The smart persistence and persistence models both converge as the forecast horizon decreases. 

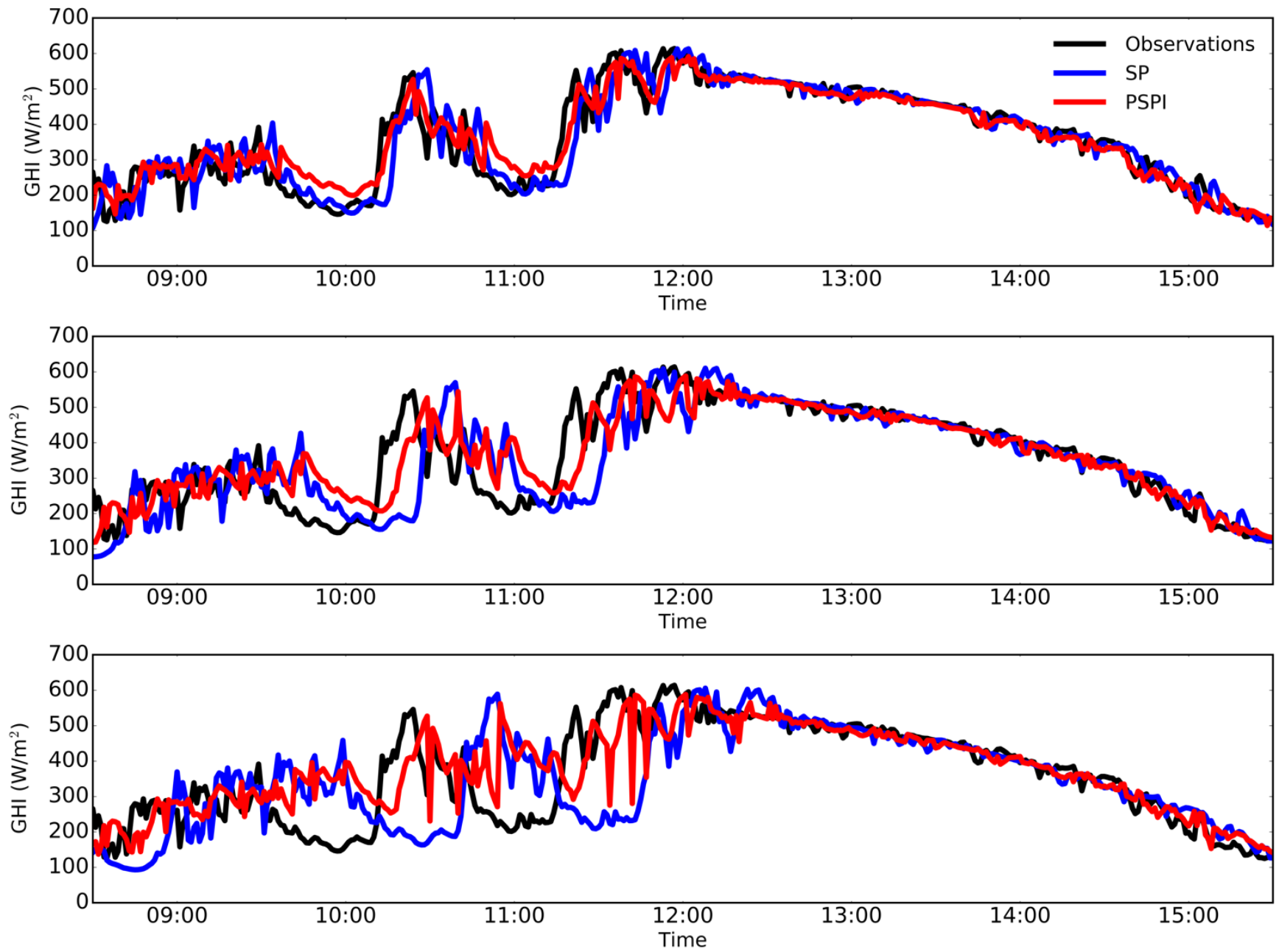

Figure 2. Five-minute (top), 15-minute (middle), and 30-minute forecast (bottom) for the smart persistence and PSPI models for the cloudy-sky day of December 2, 2013 

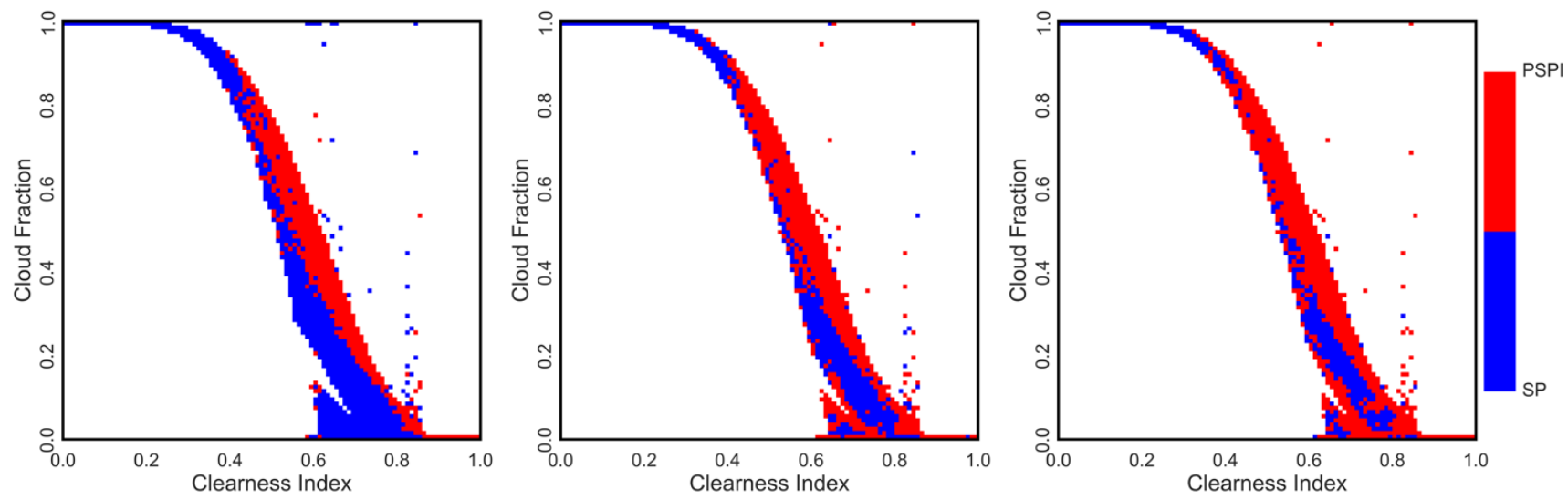

Figure 3. Clearness cloud fraction bins for the 5-minute (left), 15-minute (middle), and 30-minute (right) forecasts
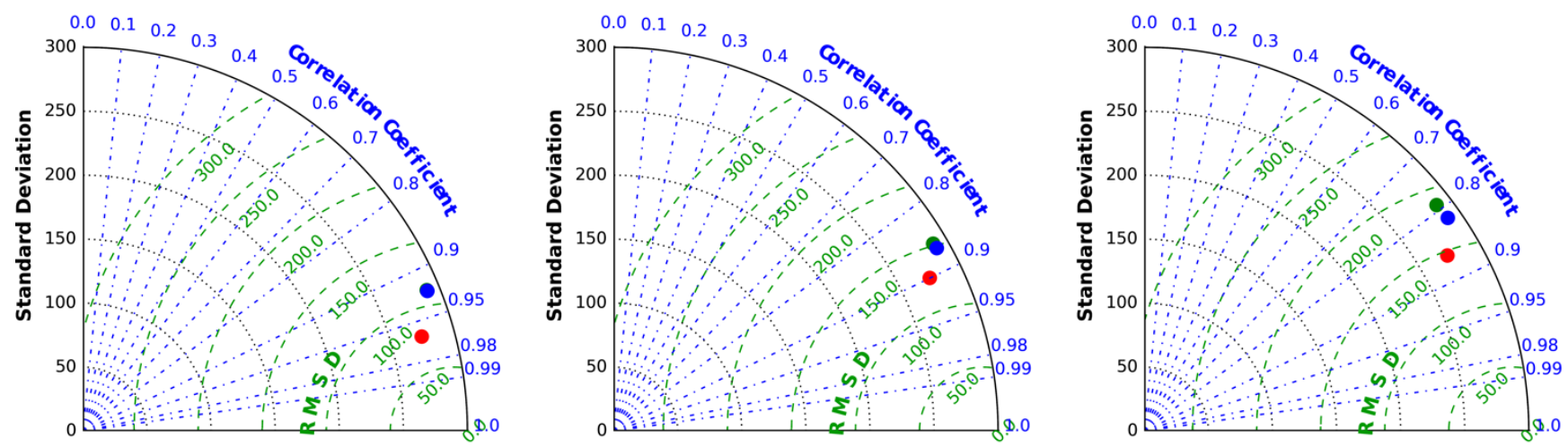

Figure 4. Taylor diagram showing the performance of the 5-minute (left), 15-minute (middle), and 30-minute (right) forecasts for the year 2013

Finally, the annual statistics for 2013 for the 5-minute, 15-minute, and 30-minute forecasts are shown in tables 1, 2, and 3 respectively. Here, the correlation coefficient, MBE, MAE, RMSE, and forecast score are displayed. Starting with the 5-minute forecast (Table 1), across the board it is evident that PSPI outperforms the smart persistence and persistence models, though this is not immediately clear by investigating only figures 2 and 3. At this forecast horizon, the models should nearly converge, which does occur for the smart persistence and persistence models. For the 15-minute forecast (Table 2), the statistics degrade some across all models, which is to be expected, but the PSPI still outperforms the other two models. The 30-minute forecast (Table 3) continues the same trend from the 15-minute forecast, but with an interesting discrepancy: the forecast score for the PSPI actually increases and is higher than the 15-minute forecast score. This primarily means that in relation to the normalized root mean square error (nRMSE) of the persistence model, the increase in nRMSE for the PSPI did not change as much when going from the 15-minute to the 30-minute forecast. This could be because of an inherent drop-off in the forecast ability for the persistence model when moving from the 15-minute to the 30-minute forecast (e.g., rate of changing cloud conditions) and/or the ability of the PSPI to still accurately capture cloud ramps despite being on a 30-minute forecast horizon. 
Table 1. Annual Statistics (2013) for the 5-Minute Forecast

\begin{tabular}{c|ccccc|} 
MODEL & CORRELATION & MBE & MAE & RMSE & $\begin{array}{c}\text { Forecast } \\
\text { Score }\end{array}$ \\
\hline PSPI & 0.96 & 10.1 & 40.0 & 78.9 & 0.30 \\
SMART PERSIST & 0.93 & 0.87 & 46.9 & 111.6 & 0.01 \\
PERSISTENCE & 0.93 & -0.17 & 50.8 & 112.3 & 0
\end{tabular}

Table 2. Annual Statistics (2013) for the 15-Minute Forecast

\begin{tabular}{c|ccccc} 
MODEL & CORRELATION & MBE & MAE & RMSE & $\begin{array}{c}\text { Forecast } \\
\text { Score }\end{array}$ \\
\hline PSPI & 0.9 & 15.1 & 65.4 & 127.8 & 0.16 \\
SMART PERSIST & 0.87 & 2.6 & 71.4 & 147.7 & 0.03 \\
PERSISTENCE & 0.87 & -0.5 & 84.6 & 151.7 & 0
\end{tabular}

Table 3. Annual Statistics (2013) for the 30-Minute Forecast

\begin{tabular}{c|ccccc|} 
MODEL & CORRELATION & MBE & MAE & RMSE & $\begin{array}{c}\text { Forecast } \\
\text { Score }\end{array}$ \\
\hline PSPI & 0.87 & 22.3 & 78.8 & 148.2 & 0.21 \\
SMART PERSIST & 0.82 & 5.23 & 91.1 & 174.3 & 0.07 \\
PERSISTENCE & 0.82 & -0.9 & 119.2 & 186.5 & 0 \\
\hline
\end{tabular}




\section{Conclusions}

For short forecast horizons where limited observations are available, and where physical aspects of the atmosphere are desired, it is beneficial to use a physical forecast model that can handle both of these situations. One of the most important situations is rapidly changing cloud conditions, which the PSPI handles better than the other persistence models. The speed of making the forecast is another concern because short-term forecasts need to update frequently to know the latest cloud conditions. Although the PSPI model does achieve these goals, some assumptions about the atmosphere in a future time period are made to achieve efficiency. Despite this, the PSPI outperforms the other persistence models on all the forecast horizons addressed in this study. The smart persistence and persistence models have been great base models for the solar energy community, with the advantages of being simple, fast, and fairly accurate for intrahour forecasts. With these forecasts, though, one forgoes the knowledge of the atmosphere, and with it cloud conditions remain unknown. In addition, GHI observations are still needed to run the smart persistence model, so it is prudent to get as much information out of the GHI observations as possible to create a better forecast (PSPI).

The PSPI model is designed to be part of a bigger grid integration software platform that produces fast ( $<1 \mathrm{~min}$ ) and reliable forecasts for grid operators and other stakeholders to use (Melton et al. 2018). In addition, the code that PSPI is built on is customizable in case more observations are available (almost always better) than the bare minimum (GHI observations). It is quite possible that the performance of the PSPI could be improved if more observations are added, but one of the main goals of this study was to see if a fast yet accurate solar forecasting model could be made with minimal observational input.

Future work is planned for the PSPI. It will be an ongoing effort because the PSPI will be opensource code on GitHub. Users can contribute anything from code improvements to better physics/models to increase the accuracy of the PSPI. Ultimately, this software is designed to help utilities and the like with their short-term forecasting needs when other methods are impractical. 


\section{References}

Benesty, J., J. Chen, Y. Huang, and L. Cohen. 2009. “Pearson Correlation Coefficient.” In Noise Reduction in Speech Processing. Heidelberg, Germany: Springer-Verlag.

Denholm, P., and M. Hand. 2011. “Grid Flexibility and Storage Required to Achieve Very High Penetration of Variable Renewable Electricity.” Energy Policy 39 (3): 1,817-1,830.

Erbs, D., S. Klein, and J. Duffie. 1982. "Estimation of the Diffuse Radiation Fraction for Hourly, Daily and Monthly-Average Global Radiation.” Solar Energy 28 (4): 293-302.

Goh, T., and K. Tan. 1977. "Stochastic Modeling and Forecasting of Solar Radiation Data." Solar Energy 19: 755-757.

Gueymard, C.A. 2014. “A Review of Validation Methodologies and Statistical Performance Indicators for Modeled Solar Radiation Data: Towards a Better Bankability of Solar Projects.” Renewable and Sustainable Energy Reviews 39: 1,024-1,034.

Haupt, S.E., B. Kosović, T. Jensen, J.K. Lazo, J.A. Lee, P.A. Jiménez, J. Cowie, G. Wiener, T.C. McCandless, and M. Rogers. 2018. "Building the Sun4Cast System: Improvements in Solar Power Forecasting.” Bulletin of the American Meteorological Society 99 (1): 121-136.

Haupt, S.E., J. Copeland, W.Y.Y. Cheng, Y. Zhang, C. Ammann, and P. Sullivan. 2016. “A Method to Assess the Wind and Solar Resource and to Quantify Interannual Variability over the United States under Current and Projected Future Climate.” Journal of Applied Meteorology and Climatology 55 (2): 345-363.

Holmgren, W.F., R.W. Andrews, A.T. Lorenzo, and J.S. Stein. 2015. "PVLIB python 2015.” Proceedings of the 2015 IEEE 42 ${ }^{\text {nd }}$ Photovoltaic Specialist Conference (PVSC).

Ineichen, P. 2016. "Validation of Models That Estimate the Clear-Sky Global and Beam Solar Irradiance.” Solar Energy 132: 332-344.

Ineichen, P., and R. Perez. 2002. “A New Airmass Independent Formulation for the Linke Turbidity Coefficient.” Solar Energy 73 (3): 151-157.

Inman, R.H., H.T. Pedro, and C.F. Coimbra. 2013. "Solar Forecasting Methods for Renewable Energy Integration.” Progress in Energy and Combustion Science 39 (6): 535-576.

Kaur, A., L. Nonnenmacher, H.T. Pedro, and C.F. Coimbra. 2016. "Benefits of Solar Forecasting for Energy Imbalance Markets.” Renewable Energy 86: 819-830.

Kleissl, J. 2013. Solar Energy Forecasting and Resource Assessment. Cambridge, MA: Academic Press.

Kuszamaul, S., A. Ellis, J. Stein, and L. Johnson. 2010. “Lanai High-Density Irradiance Sensor Network for Characterizing Solar Resource Variability of MW-Scale PV System.” Proceedings of the 2010 35 $5^{\text {th }}$ IEEE Photovoltaic Specialists Conference. 
Marquez, R., and C.F. Coimbra. 2013. "Proposed Metric for Evaluation of Solar Forecasting Models.” Journal of Solar Energy Engineering 135 (1): 011016.

Mathiesen, P., and J. Kleissl. 2011. "Evaluation of Numerical Weather Prediction for Intra-Day Solar Forecasting in the Continental United States.” Solar Energy 85 (5): 967-977.

Meador, W., and W. Weaver. 1980. “Two-Stream Approximations to Radiative Transfer in Planetary Atmospheres: A Unified Description of Existing Methods and a New Improvement.” Journal of the Atmospheric Sciences 37 (3): 630-643.

Melton, R.B., K.P. Schneider, E. Lightner, T.E. McDermott, P. Sharma, Y. Zhang, F. Ding, S. Vadari, R. Podmore, and A. Dubey. 2018. "Leveraging Standards to Create an Open Platform for the Development of Advanced Distribution Applications.” IEEE Access 6: 37,361-37,370.

Mills, A., Botterud, A., Wu, J., Zhou, Z., Hodge, B.M., and Heaney, M. 2013. Integrating Solar PV in Utility System Operations. (ANL/DIS-13/18). Lemont, IL: Argonne National Laboratory.

Palmintier, B., R. Broderick, B. Mather, M. Coddington, K. Baker, F. Ding, M. Reno, M. Lave, and A. Bharatkumar. 2016. On the Path to SunShot: Emerging Issues and Challenges in Integrating Solar with the Distribution System (NREL/TP-5D00-65331). Golden, CO: National Renewable Energy Laboratory.

Perez, R., E. Lorenz, S. Pelland, M. Beauharnois, G. Van Knowe, K. Hemker Jr, D. Heinemann, J. Remund, S.C. Müller, and W. Traunmüller. 2013. "Comparison of Numerical Weather Prediction Solar Irradiance Forecasts in the US, Canada, and Europe.” Solar Energy 94: 305326.

Reno, M.J., C.W. Hansen, and J.S. Stein. 2012. Global Horizontal Irradiance Clear-Sky Models: Implementation and Analysis (SAND2012-2389). Albuquerque, NM: Sandia National Laboratories.

Ruiz-Arias, J., H. Alsamamra, J. Tovar-Pescador, and D. Pozo-Vázquez. 2010. "Proposal of a Regressive Model for the Hourly Diffuse Solar Radiation Under All-Sky Conditions.” Energy Conversion and Management 51 (5): 881-893.

Sagan, C., and J.B. Pollack. 1967. "Anisotropic Nonconservative Scattering and the Clouds of Venus.” Journal of Geophysical Research 72 (2): 469-477.

Sengupta, M., A. Habte, C. Gueymard, S. Wilbert, and D. Renne. 2017. Best Practices Handbook for the Collection and Use of Solar Resource Data for Solar Energy Applications (NREL/TP-5D00-68886). Golden, CO: National Renewable Energy Laboratory.

Stoffel, T., and A. Andreas. 1981. "NREL Solar Radiation Research Laboraotry (SRRL): Baseline Measurement System (BMS).” (NREL/DA-5500-56488). Golden, CO: National Renewable Energy Laboratory. 
Taylor, K.E. 2001. "Summarizing Multiple Aspects of Model Performance in a Single Diagram.” Journal of Geophysical Research: Atmospheres 106 (D7): 7,183-7,192.

Xie, Y., and Y. Liu. 2013. "A New Approach for Simultaneously Retrieving Cloud Albedo and Cloud Fraction from Surface-Based Shortwave Radiation Measurements.” Environmental Research Letters 8 (4): 044023.

Yang, D., J. Kleissl, C.A. Gueymard, H.T. Pedro, and C.F. Coimbra. 2018. "History and Trends in Solar Irradiance and PV Power Forecasting: A Preliminary Assessment and Review Using Text Mining.” Solar Energy. 